\title{
The Marshall-Olkin Generalized Inverse Weibull Distribution: Properties and Application
}

\author{
Hamdy M. Salem ${ }^{1}$ \\ ${ }^{1}$ School Department of Statistics, Faculty of Commerce, Al-Azher University, Egypt \&Community College in \\ Buraidah, Qassim University, Saudi Arabia \\ Correspondence: Hamdy M. Salem, School Department of Statistics, Faculty of Commerce, Al-Azher University, \\ Egypt \&Community College in Buraidah, Qassim University, Saudi Arabia. E-mail: d.hamdysalm@yahoo.com
}

Received: Dec.6, 2018

doi:10.5539/mas.v13n2p54
Accepted: Dec.17, $2018 \quad$ Online Published: January 3, 2019

URL: https://doi.org/10.5539/mas.v13n2p54

\begin{abstract}
In this paper, a new distribution namely, The Marshall-OlkinGeneralized Inverse Weibull Distribution is illustrated and studied. The new distribution is very flexible and contains sub-models such asinverse exponential, inverse Rayleigh, Weibull, inverse Weibull, Marshall-Olkininverse Weibull and Fréchetdistributions. Also, the hazard function of the new distribution can produce variety of forms:an increase, a decrease and an upside-down bathtub. Some properties such as hazard function, quintile function, entropy, moment generating function and order statistics are obtained. Different estimation approaches namely, maximum likelihood estimators, interval estimators, least square estimators, fisher information matrix and asymptotic confidence intervals are described. To illustrate the superior performance of the proposed distribution, a simulation study and a real data analysis are investigated against other models.
\end{abstract}

Keywords: Marshall-Olkin family, Inverse Weibull Distribution, Maximum likelihood estimator, Hazard function, Order Statistics

\section{Introduction}

Recently, many authors have been interested in the Weibull distribution which is extensively used over the past decades for reliability studies and is adequate for modeling monotone data. In some studies, such breast cancer data and mortality of lung, many authors showed that the Inverse Weibull IW distribution is an appropriate model for this kind of data because its hazard function is non-monotone or unimodal (see for example (Bennett 1983), (Langlands 1979), (Kundu and Howladu 2010)). Several authors studied this distribution in different fields as (Drapella 1993), (Mudholkar and Kollia 1994).

By adding a new shape parameter to the distribution function of the IW distribution, the new distribution is called the Generalized Inverse Weibull GIW distribution. (Felipe et al. 2011) discussed the properties of the GIW distribution including hazard function, survival function, central and non-central moments and moment generating function. They provided maximum likelihood estimators, asymptotic confidence intervals and fisher information matrix as well. The cumulative density function cdf and probability density function pdf of the GIW distribution respectively are

$$
F(x)=e^{-\lambda\left(\frac{\alpha}{x}\right)^{\beta}} \quad ; \quad x, \alpha, \beta, \lambda>0
$$

And

Where $\beta$ and $\lambda$ are the shape parameters.

$$
f(x)=\lambda \beta \alpha^{\beta} x^{-(\beta+1)} e^{-\lambda\left(\frac{\alpha}{x}\right)^{\beta}} \quad ; \quad x, \alpha, \beta, \lambda>0
$$

In the recent past, to analyze complex data and obtaining a better fit, researchers were interested in generating new continuous distributions. Also, comprehensive studies used Marshall-Olkin method in generating new distributions. Some statistical properties of these new distributions were illustrated. For example, Marshall-Olkin logistic processes has been introduced by (Alice and Jose 2005), (Gui 2013) studied Marshall-Olkin power lognormal distribution. Marshall-Olkin extended Weibull distribution was studied by (Cordeiro and Lemonte 
2013). (Ghitany et al. 2007) introduced Marshall-Olkin Extended Lomax distribution.

(Marshall and Olkin 1997) have added a parameter to the base line cdf in exponential and Weibull families as follows

$$
G(x)=\frac{F(x)}{\theta-(\theta-1) F(x)} \quad ; \quad 0<x<\infty, \theta>0
$$

This study aims at providing a new extension of weibull distribution namely, The Marshall-Olkin Generalized Inverse Weibull (MOGIW for short) distribution. It contains more significant fits than some distributions such as Marshall-Olkin extended inverse Weibull, inverse Weibull, Fréchet, inverse Rayleigh and inverse exponential being sub-models from the proposed distribution.

The motivations to introduce the MOGIW distribution are: (i) some known distributions are sub-models from it; (ii) in case the hazard function is non-monotone or unimodal, the MOGIW distribution is a superior fit to other common lifetime distributions; (iii) the MOGIW distribution can use in different areas such as breast cancer and wear-out periods in medical and physical fields respectively; (iv) to illustrate the applicability of the MOGIW distribution by conducting study of a numerical and an actual data.

This paper is organized as follows: the model definition and its statistical properties including hazard function, quintile function, moments, moment generating function and order statistics are discussed in section 2 . Then, different estimation approaches namely, maximum likelihood estimators, asymptotic confidence intervals based on asymptotic normality, fisher information matrix, interval estimators and least square estimators are described in section 3. Finally, in section 4, a simulation study and a real date set are conducted to illustrate the performance of the proposed distribution.

\section{New Model and its Properties}

in this section, the cdf and pdf for the proposed distribution and some of its statistical properties are discussed. Let $\mathrm{X}$ be a random variable with cdf of the GIW distribution which in (1) and by substitution in (3), then, the cdf of the MOGIW distribution is given as

$$
G(x)=\frac{e^{-\lambda\left(\frac{\alpha}{x}\right)^{\beta}}}{\theta-(\theta-1) e^{-\lambda\left(\frac{\alpha}{x}\right)^{\beta}}} ; \quad x, \alpha, \beta, \lambda, \theta>0
$$

and the corresponding pdf is

$$
g(x)=\frac{\lambda \beta \theta \alpha^{\beta} x^{-(\beta+1)} e^{-\lambda\left(\frac{\alpha}{x}\right)^{\beta}}}{\left[\theta-(\theta-1) e^{-\lambda\left(\frac{\alpha}{x}\right)^{\beta}}\right]^{2}} \quad ; x, \alpha, \beta, \lambda, \theta>0
$$

Figure 1 and 2 describe different forms to the pdf and cdf respectively of the MOGIW distribution at different values for the parameters $\alpha, \beta, \theta$ and $\lambda$.

The distribution which in (4) has special cases as the following

(i) If $\lambda=1$, then, the MOGIW distribution reduces to the Marshall-Olkin extended inverse Weibull distribution which studied by (Okasha et al 2017) with parameters $\beta, \theta$ and $\alpha$.

(ii) If $\theta=\beta=1$, then, it reduces to the exponentiated Inverse Weibull distribution.

(iii) If $\lambda=\theta=1$, then, the MOGIW distribution reduces to the Inverse Weibull distribution.

(iv) If $\theta=1, \beta=2$ and $\lambda=1$, then, it reduces to the Inverse Rayleigh distribution.

(v) If $\beta=\theta=1$ and $\lambda=1$, then, it reduces to the Inverse exponential distribution. 


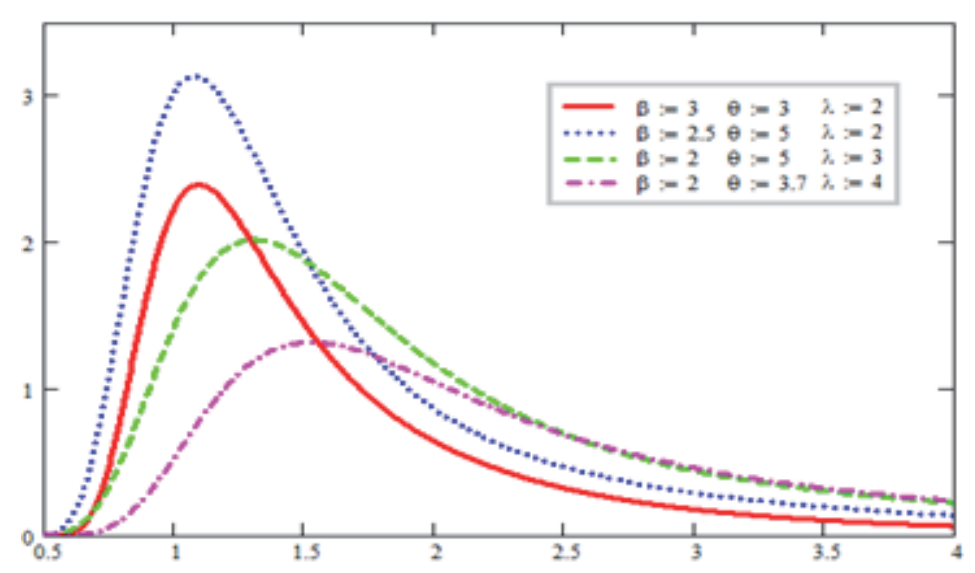

Figure 1. The pdf forms of the MOGIW distribution at $\alpha=1$ and different values of the parameters $\beta, \theta$ and $\lambda$

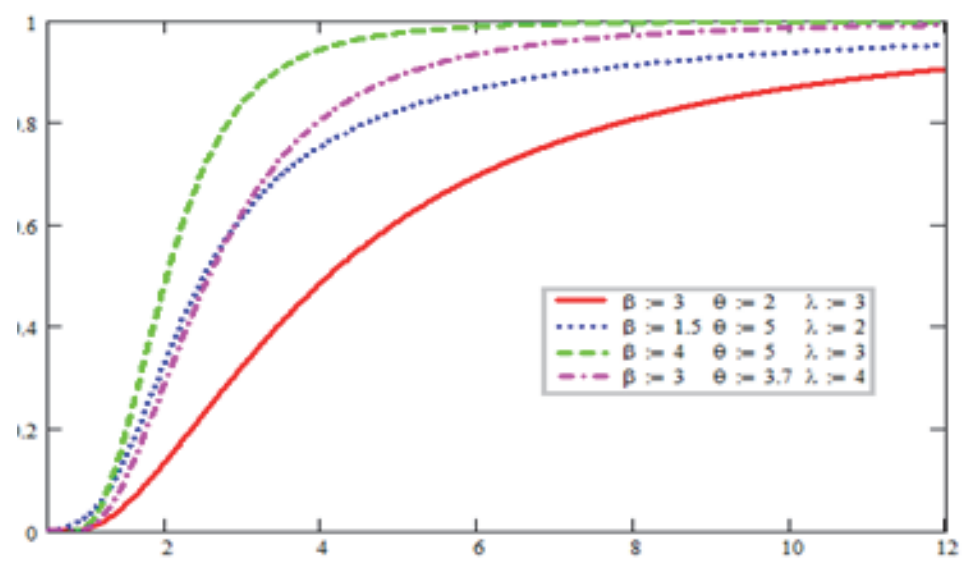

Figure 2. The cdf forms of the MOGIW distribution at $\alpha=1$ and different values of the parameters $\beta, \theta$ and Using the expansion $(1-z)^{-k}=\sum_{i=0}^{\infty} \frac{\Gamma(k+1) z^{i}}{\Gamma(k) i !}$ where $|z|<1, k>0$ and $\Gamma($.$) is a gamma function, then,$ the equations (4) and (5) can be written respectively as

$$
G(x)=\theta^{2} \sum_{i=0}^{\infty}(i+1)^{-1}\left(\frac{\theta}{\theta-1}\right)^{i} e^{-\lambda(i+1)\left(\frac{\alpha}{x}\right)^{\beta}}
$$

and

$$
g(x)=\lambda \beta \theta^{3} \alpha^{\beta} \sum_{i=0}^{\infty}(i+1)^{-1}\left(\frac{\theta}{\theta-1}\right)^{i} x^{-(\beta+1)} e^{-\lambda(i+1)\left(\frac{\alpha}{x}\right)^{\beta}}
$$

The survival function $\mathrm{R}(\mathrm{x})$ and the hazard rate function $\mathrm{h}(\mathrm{x})$ of the MOGIW distribution respectively are

$$
R(x)=1-\frac{e^{-\lambda\left(\frac{\alpha}{x}\right)^{\beta}}}{\theta-(\theta-1) e^{-\lambda\left(\frac{\alpha}{x}\right)^{\beta}}}
$$

and 


$$
h(x)=\frac{f(x)}{R(x)}=\frac{\lambda \beta \theta \alpha^{\beta} x^{-(\beta+1)} e^{-\lambda\left(\frac{\alpha}{x}\right)^{\beta}}}{\left[\theta\left(e^{-\lambda\left(\frac{\alpha}{x}\right)^{\beta}}-\theta\right)-e^{-\lambda\left(\frac{\alpha}{x}\right)^{\beta}}\right] \cdot\left[e^{-\lambda\left(\frac{\alpha}{x}\right)^{\beta}}-1\right]}
$$

\subsection{Moments}

In any statistical analysis, moments are essential, important and helpful in applications. The $\mathrm{r}^{\text {th }}$ moment $\mu_{r}^{\prime}$ of the MOGIW distribution is

$$
\begin{array}{r}
E(X)^{r}=\int_{0}^{\infty} x^{r} g(x) d x=\frac{\beta \alpha^{\beta}}{\theta} \sum_{i=0}^{\infty}(i+1)\left(\frac{\theta-1}{\theta}\right)^{i} \int_{0}^{\infty} x^{r-(\beta+1)} e^{-\lambda(i+1)\left(\frac{\alpha}{x}\right)^{\beta}} d x \\
=\frac{1}{\theta} \alpha^{r+1} \sum_{i=0}^{\infty}(i+1)\left(\frac{\theta-1}{\theta}\right)^{i}[\lambda(i+1)]^{\frac{r}{\beta}+1} \Gamma\left(1-\frac{r}{\beta}\right)
\end{array}
$$

When $r$ equal one, two, three and four in (10), the $\mathrm{J}^{\text {st }}$ four moments for MOGIW distribution are given respectively. Also, by using the relation between the central and non-central moments which is

$\mu_{r}=E(X-\mu)^{r}=\sum_{j=0}^{\infty}\left(\begin{array}{l}r \\ j\end{array}\right)(-1)^{j-1} \mu_{1}^{\prime} \mu^{r-j}$, then, the central moments $\mu_{r}$ are computed.

\subsection{Quantile Function and Median}

By inverting (4) and suppose that $Q(\eta), 0<\eta<1$ denote the quantile function of the MOGIW distribution, then, $Q(\eta)$ is given by

$$
x_{u}=\alpha \sqrt[-\beta]{\frac{\ln \left(\frac{\eta \theta-\eta+1}{\eta \theta}\right)}{\lambda}}
$$

Setting $\eta=(0.25,0.5,0.75)$ in (11), then, the 1 st, 2 nd and 3rd quartiles of the MOGIW distribution can be obtained. So, the median is

$$
x_{0.5}=\alpha \sqrt[-\beta]{\frac{\ln \left(\frac{0.5 \theta+0.5}{0.5 \theta}\right)}{\lambda}}
$$

\subsection{Moment Generating Function}

From (9), the moment generating function can be obtained as follows

$$
\begin{aligned}
M(t)=E\left(e^{t x}\right) & =\int_{0}^{\infty} e^{t x} g(x) d x \\
& =\frac{\beta \alpha^{\beta}}{\theta} \sum_{i=0}^{\infty}(i+1)\left(\frac{\theta-1}{\theta}\right)^{i} \int_{0}^{\infty} x^{-(\beta+1)^{i}} e^{t x-\lambda(i+1)\left(\frac{\alpha}{x}\right)^{\beta}} d x
\end{aligned}
$$

\subsection{Order statistics}

In statistical theory and application fields, the order statistics are appeared. In a random sample of size $\mathrm{n}$ from the MOGIW distribution, the density function $f_{r: n}(x)$ of the $j^{\text {th }}$ order statistic $X_{r: n}$ can be written as 


$$
\begin{aligned}
f_{r: n}(x) & =\frac{1}{\beta(r, n-r+1)} g(x)\left[G^{r-1}(x)\right][1-G(x)]^{n-r} \\
& =\frac{1}{\beta(r, n-r+1)} \sum_{k=0}^{\infty}(-1)^{k}\left(\begin{array}{c}
n-r \\
k
\end{array}\right) g(x)[G(x)]^{k+r-1}
\end{aligned}
$$

\section{Parameter Estimations}

This section concerns with the estimation of parameters for MOGIW distribution using maximum likelihood estimator MLEs and least square estimator LSs methods. Also, fisher information matrix and asymptotic confidence intervals are discussed.

\subsection{Maximum liklihood estimators}

Let $x_{1}, x_{2}, \ldots, x_{n}$ be a random sample from MOGIW distribution $X \approx \operatorname{MOGIW}(\underline{\tau})$, then, the likelihood and log-likelihood functions for $\underline{\tau}=(\lambda, \beta, \theta, \alpha)$ can be written respectively as

$$
L=\frac{\left(\lambda \beta \theta \alpha^{\beta}\right)^{n} \prod_{j=1}^{n} x_{j}^{-(\beta+1)} \prod_{j=1}^{n} e^{-\lambda\left(\frac{\alpha}{x_{j}}\right)^{\beta}}}{\prod_{j=1}^{n}\left[\theta-(\theta-1) e^{-\lambda\left(\frac{\alpha}{x_{j}}\right)^{\beta}}\right]^{2}}
$$

and

$$
\begin{aligned}
\ell=n \ln \left(\lambda \beta \theta \alpha^{\beta}\right)-(\beta+1) \sum_{j=1}^{n} & \ln \left(x_{j}\right)-\lambda\left(\frac{\alpha}{x_{j}}\right)^{\beta} \\
& -2 \sum_{j=1}^{n} \ln \left(\theta-(\theta-1) e^{-\lambda\left(\frac{\alpha}{x_{j}}\right)^{\beta}}\right)
\end{aligned}
$$

The first derivative of $\ell$ for the parameters $\lambda, \beta, \theta$ and $\alpha$ respectively are

$$
\begin{gathered}
\frac{\partial \ell}{\partial \alpha}=n \lambda \beta^{2} \theta \alpha^{\beta-1}-\frac{\lambda \beta}{\alpha}\left(\frac{\alpha}{x_{j}}\right)^{\beta}+\frac{2(\theta-1) \lambda \beta}{\alpha}\left(\frac{\alpha}{x_{j}}\right)^{\beta} \\
\times \sum_{j=1}^{n} s \\
\frac{\partial \ell}{\partial \beta}=n \lambda \beta \theta \alpha^{\beta} \ln (\alpha)+n \lambda \theta \alpha^{\beta}-\sum_{j=1}^{n} \ln \left(x_{j}\right)-\lambda\left(\frac{\alpha}{x_{j}}\right)^{\beta} \ln \left(\frac{\alpha}{x_{j}}\right) \\
+2 \lambda(\theta-1) \lambda \sum_{j=1}^{n}\left(\frac{\alpha}{x_{j}}\right)^{\beta} \ln \left(\frac{\alpha}{x_{j}}\right) s \\
\frac{\partial \ell}{\partial \theta}=n \lambda \beta \alpha^{\beta}-2 \sum_{j=1}^{n} s
\end{gathered}
$$




$$
\begin{gathered}
\frac{\partial \ell}{\partial \lambda}=n \beta \theta \alpha^{\beta}-\left(\frac{\alpha}{x_{j}}\right)^{\beta}-2(\theta-1)\left(\frac{\alpha}{x_{j}}\right)^{\beta} \sum_{j=1}^{n} s \\
\text { Where } s=\frac{e^{-\lambda\left(\frac{\alpha}{x_{j}}\right)^{\beta}}}{\left(\theta-(\theta-1) e^{\left.-\lambda\left(\frac{\alpha}{x_{j}}\right)^{\beta}\right)}\right.} \cdot
\end{gathered}
$$

By solving the last 4 nonlinear equations iteratively after equal them by zero, then, the MLEs of the parameters are found.

Now, to construct the elements of asymptotic Fisher information matrix, the second derivative of $\ell$ for the parameters $\lambda, \beta, \theta$ and $\alpha$ respectively are obtained and inverting the observed information matrix.

$$
\begin{aligned}
& \frac{\partial^{2} \ell}{\partial \alpha^{2}}=n \lambda \beta^{2}(\beta-1) \theta \alpha^{\beta-2}-\frac{\lambda \beta}{\alpha^{2}}(\beta-1)\left(\frac{\alpha}{x_{j}}\right)^{\beta}+\frac{2(\theta-2) \lambda \beta}{\alpha^{2}} \\
& \times \sum_{j=1}^{n}\left(\frac{\alpha}{x_{j}}\right)^{\beta} s\left(\beta-1-\beta \lambda\left(\frac{\alpha}{x_{j}}\right)^{\beta}(1-s)\right) \\
& \frac{\partial^{2} \ell}{\partial \beta^{2}}=n \alpha^{\beta} \ln (\alpha) \theta \lambda(\beta \ln (\alpha)+2)+\lambda\left(\frac{\alpha}{x_{j}}\right)^{\beta} \ln \left(\frac{\alpha}{x_{j}}\right)^{2} \\
& -\sum_{j=1}^{n} t s\left(1-\lambda\left(\frac{\alpha}{x_{j}}\right)^{\beta}-\lambda(\theta-1) s\left(\frac{\alpha}{x_{j}}\right)^{\beta}\right) \\
& \frac{\partial^{2} \ell}{\partial \theta^{2}}=2 \sum_{j=1}^{n}\left(\frac{1-e^{-\lambda\left(\frac{\alpha}{x_{j}}\right)^{\beta}}}{\left(\theta-(\theta-1) e^{-\lambda\left(\frac{\alpha}{x_{j}}\right)^{\beta}}\right)}\right)^{2} \\
& \frac{\partial^{2} \ell}{\partial \lambda^{2}}=2 \sum_{j=1}^{n} \frac{t s+t s^{2}}{\lambda \ln \left(\frac{\alpha}{x_{j}}\right)^{2}}
\end{aligned}
$$




$$
\begin{aligned}
& \frac{\partial^{2} \ell}{\partial \alpha \partial \beta}=n \alpha^{\beta-1} \beta \lambda(\ln (\beta) \beta+2)-\lambda\left(\frac{\alpha}{x_{j}}\right)^{\beta} \ln \left(\frac{\alpha}{x_{j}}\right) \\
& -\frac{\lambda}{\alpha}\left(\frac{\alpha}{x_{j}}\right)^{\beta}-\frac{\beta}{\alpha} \sum_{j=1}^{n}\left[\frac{t s}{\ln \left(\frac{\alpha}{x_{j}}\right)}+\frac{t s}{\ln \left(\frac{\alpha}{x_{j}}\right)^{2}}\right. \\
& \left.-\frac{t s}{\ln \left(\frac{\alpha}{x_{j}}\right)}+\frac{t s}{\ln \left(\frac{\alpha}{x_{j}}\right)^{2}}\right] \\
& \frac{\partial^{2} \ell}{\partial \alpha \partial \theta}=n \alpha^{\beta-1} \beta^{2} \lambda-\frac{\beta}{\alpha} \sum_{j=1}^{n}\left[2 \lambda\left(\frac{\alpha}{x_{j}}\right)^{\beta} s\right. \\
& \left.\left.+\frac{t s e^{-\lambda\left(\frac{\alpha}{x_{j}}\right)^{\beta}}}{\ln \left(\left(\frac{\alpha}{x_{j}}\right)\right)}\right)\right] \\
& \frac{\partial^{2} \ell}{\partial \alpha \partial \lambda}=\frac{\alpha^{\beta} \beta^{2}}{\alpha}(n \theta-\beta)-\frac{\beta}{\alpha} \sum_{j=1}^{n} \frac{1}{\ln \left(\frac{\alpha}{x_{j}}\right)^{2}}\left(\frac{t s}{\lambda}-t s-t s^{2}(\theta-1)\right) \\
& \frac{\partial^{2} \ell}{\partial \beta \partial \theta}=n \alpha^{\beta} \beta \lambda-2 \sum_{j=1}^{n} s \\
& \frac{\partial^{2} \ell}{\partial \beta \partial \lambda}=n \alpha^{\beta} \beta \theta-\left(\frac{\alpha}{x_{j}}\right)^{\beta}-2 \sum_{j=1}^{n}\left(\frac{\alpha}{x_{j}}\right)^{\beta}(\theta-1) s \\
& \frac{\partial^{2} \ell}{\partial \theta \partial \lambda}=n \alpha^{\beta} \beta-\sum_{j=1}^{n}\left(\frac{\alpha}{x_{j}}\right)^{\beta}\left[2 s-(2 \theta-2) \frac{e^{-\lambda\left(\frac{\alpha}{x_{j}}\right)^{\beta}\left(1-e^{-\lambda\left(\frac{\alpha}{x_{j}}\right)^{\beta}}\right)}}{\left(\theta-(\theta-1) e^{-\lambda\left(\frac{\alpha}{x_{j}}\right)^{\beta}}\right)^{2}}\right]
\end{aligned}
$$




$$
\text { Where } s=\frac{e^{-\lambda\left(\frac{\alpha}{x_{j}}\right)^{\beta}}}{\left(\theta-(\theta-1) e^{-\lambda\left(\frac{\alpha}{x_{j}}\right)^{\beta}}\right)} \text { and } t=\lambda(2 \theta-2)\left(\frac{\alpha}{x_{j}}\right)^{\beta} \ln \left(\frac{\alpha}{x_{j}}\right)^{2}
$$

By solving the inverse dispersion matrix analytically, then, the asymptotic variance and covariance matrix of the MLs 瓷 $\beta, \theta$ and $\hat{\alpha}$ for the parameters $\lambda, \beta, \theta$ and $\alpha$ respectively is yielded.

\subsection{Asymptotic Confidence Intervals}

To construct approximate confidence intervals for the parameters, the expected information matrix is one of the mean requirement for derive the confidence interval estimates. (Lindgren 1976) showed that for the large samples and under certain regularity conditions, the MLs say, $\hat{\tau}=($ 次, $\beta, \hat{\theta}, \hat{\alpha})$ for the parameters $\underline{\tau}=(\lambda, \beta, \theta, \alpha)$ are asymptotically normally distributed with mean $\hat{\tau}$ and variance $\sigma^{2}(\hat{\tau})$.. Now, let $x_{1}, x_{2}, \ldots, x_{n}$ be a random sample from MOGIW distribution $X \approx \operatorname{MOGIW}(\underline{\tau})$, then, the lower and upper confidence intervals for the parameters with approximate $100 \%(1-\gamma)$ percentage are

$$
\begin{array}{ll}
\hat{\alpha} \pm Z_{\gamma / 2} \sigma(\hat{\alpha}), & \hat{\beta} \pm Z_{\gamma / 2} \sigma(\hat{\beta}), \\
\hat{\theta} \pm Z_{\gamma / 2} \sigma(\hat{\theta}), & \hat{\lambda} \pm Z_{\gamma / 2} \sigma(\hat{\lambda})
\end{array}
$$

\subsection{Least Square Estimators}

Swain et al. (1988) [20] used an originally least-square method to parameter estimations for Beta distributions. This method depends on minimizing the distance between the vector of order statistics and its corresponding expected values. Let $x_{1}, x_{2}, \ldots, x_{n}$ be a random sample of size $\mathrm{n}$ from the MOGIW distribution function $G\left(X_{i}\right)$ and $x_{(1)}, x_{(2)}, \ldots, x_{(n)}$ are order statistics of the observed sample $E\left[G\left(X_{(i)}\right)\right]=\frac{i}{n+1}$, then, the least-square estimator $\tilde{\tau}=(\lambda, \beta, \theta, \widetilde{\alpha})$ for the parameters $\underline{\tau}=(\lambda, \beta, \theta, \alpha)$ can obtained by differentiate the following equation with respect to $\lambda, \beta, \theta$ :and $\alpha$ :

$$
\sum_{i=1}^{n}\left(\frac{e^{-\lambda\left(\frac{\alpha}{x_{(i)}}\right)^{\beta}}}{\theta-(\theta-1) e^{-\lambda\left(\frac{\alpha}{x_{(i)}}\right)^{\beta}}-\frac{i}{n+1}}\right)^{2}=0
$$

So, (6) gives 


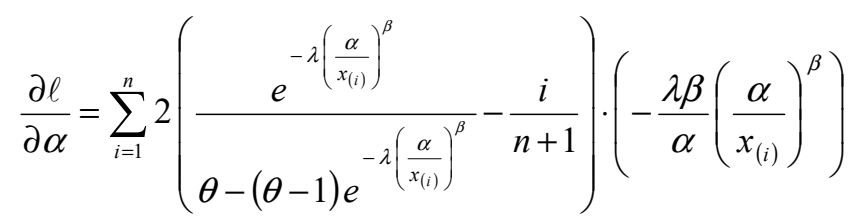

$$
\begin{aligned}
& \times\left(\frac{e^{-\lambda\left(\frac{\alpha}{x_{(i)}}\right)^{\beta}}+(\theta-1)}{\theta-(\theta-1) e^{-\lambda\left(\frac{\alpha}{x_{(i)}}\right)^{\beta}}}\left[\frac{e^{-\lambda\left(\frac{\alpha}{x_{(i)}}\right)^{\beta}}}{\theta-(\theta-1) e^{-\lambda\left(\frac{\alpha}{x_{(i)}}\right)^{\beta}}}\right]^{2}\right)
\end{aligned}
$$

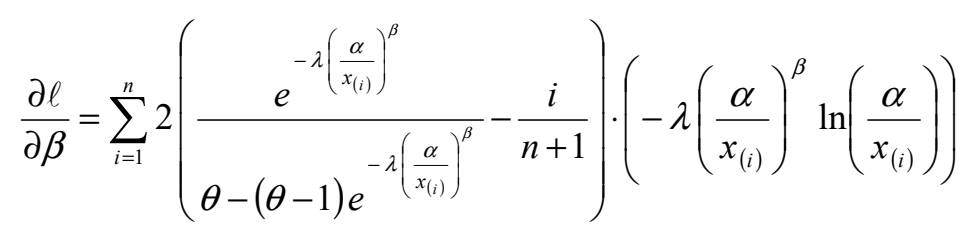

$$
\begin{aligned}
& \times\left(\frac{e^{-\lambda\left(\frac{\alpha}{x_{(i)}}\right)^{\beta}}}{\theta-(\theta-1) e^{-\lambda\left(\frac{\alpha}{x_{(i)}}\right)^{\beta}}+(\theta-1)}\left[\frac{e^{-\lambda\left(\frac{\alpha}{x_{(i)}}\right)^{\beta}}}{\theta-(\theta-1) e^{-\lambda\left(\frac{\alpha}{x_{(i)}}\right)^{\beta}}}\right]^{2}\right) \\
& \frac{\partial \ell}{\partial \theta}=\sum_{i=1}^{n}-2\left(\frac{e^{-\lambda\left(\frac{\alpha}{x_{(i)}}\right)^{\beta}}}{\theta-(\theta-1) e^{-\lambda\left(\frac{\alpha}{x_{(i)}}\right)^{\beta}}-\frac{i}{n+1}}\right) \cdot \frac{\left.e^{-\lambda\left(\frac{\alpha}{x_{(i)}}\right)^{\beta}}\left(1-e^{-\lambda\left(\frac{\alpha}{x_{(i)}}\right)^{\beta}}\right)^{-\lambda\left(\frac{\alpha}{x_{(i)}}\right)^{\beta}}\right]^{2}}{\left[\theta-(\theta-1) e^{2}\right.} \\
& \frac{\partial \ell}{\partial \lambda}=\sum_{i=1}^{n} 2\left(\frac{e^{-\lambda\left(\frac{\alpha}{x_{(i)}}\right)^{\beta}}}{\theta-(\theta-1) e^{-\lambda\left(\frac{\alpha}{x_{(i)}}\right)^{\beta}}-\frac{i}{n+1}}\right) \cdot\left(-\left(\frac{\alpha}{x_{(i)}}\right)^{\beta}\right)
\end{aligned}
$$

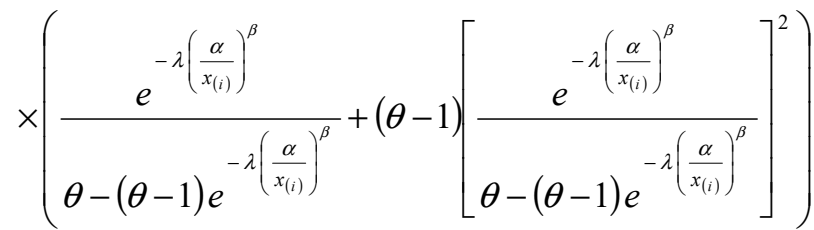

By solving the last four system equations in $\lambda, \beta, \theta$ and $\alpha$ numerically, then, the least square estimators can be obtained.

\section{Data Analysis with Numerical Comparisons and Empirical Application}

In this section, a simulation study and real date set are conducted to illustrate the performance of the proposed distribution. To obtain a numerical illustration for the last theoretical results, MATHCAD software is used. 1000 samples generated from MOGIW distribution with parameters $\lambda, \beta, \theta$ and $\alpha$ at various size samples. MLEs, Relative bias (R Bias) and mean square error (MSE) are evaluated for the MOGIW distribution parameters.

Where R Bias $=\left|\frac{\hat{\varepsilon}-\varepsilon}{\varepsilon}\right|$ and MSE $=E(\hat{\varepsilon}-\varepsilon)^{2}$. They are included in table (1) and them values are decreasing 
by increasing the sample size $n$.

Table 1. MLES, R BIAS and MSE of parameters estimates

\begin{tabular}{|c|c|c|c|c|c|c|c|c|c|c|c|c|c|c|c|c|}
\hline \multirow{2}{*}{$\alpha$} & \multirow{2}{*}{$\beta$} & \multirow{2}{*}{$\theta$} & \multirow{2}{*}{$\lambda$} & \multirow[b]{2}{*}{$\mathrm{n}$} & \multicolumn{3}{|l|}{$\hat{\alpha}$} & \multicolumn{3}{|l|}{$\beta$} & \multicolumn{3}{|l|}{$\theta$} & \multicolumn{3}{|l|}{$\lambda$} \\
\hline & & & & & MLE & $\begin{array}{c}\mathrm{R} \\
\text { Bias }\end{array}$ & MSE & MLE & $\begin{array}{l}\mathrm{R} \\
\text { Bias }\end{array}$ & MSE & MLE & $\begin{array}{c}\mathrm{R} \\
\text { Bias }\end{array}$ & MSE & MLE & $\begin{array}{l}\mathrm{R} \\
\text { Bias }\end{array}$ & MSE \\
\hline \multirow{5}{*}{0.5} & \multirow{5}{*}{1} & \multirow{5}{*}{0.5} & \multirow{5}{*}{2} & 25 & $\begin{array}{l}0.48 \\
7\end{array}$ & -0.010 & 0.010 & $\begin{array}{l}0.99 \\
0\end{array}$ & -0.010 & 0.010 & $\begin{array}{l}0.43 \\
3\end{array}$ & -0.010 & 0.010 & $\begin{array}{l}1.98 \\
7\end{array}$ & -0.013 & 0.014 \\
\hline & & & & 40 & $\begin{array}{l}0.49 \\
1\end{array}$ & -0.010 & 0.010 & $\begin{array}{l}0.99 \\
0\end{array}$ & -0.010 & 0.010 & $\begin{array}{l}0.46 \\
9\end{array}$ & -0.011 & 0.010 & $\begin{array}{l}1.99 \\
1\end{array}$ & -0.013 & 0.014 \\
\hline & & & & $\begin{array}{l}10 \\
0\end{array}$ & $\begin{array}{l}0.49 \\
0\end{array}$ & -0.010 & 0.010 & $\begin{array}{l}0.99 \\
1\end{array}$ & -0.009 & 0.010 & $\begin{array}{l}0.48 \\
2\end{array}$ & -0.018 & 0.009 & $\begin{array}{l}1.99 \\
0\end{array}$ & -0.013 & 0.014 \\
\hline & & & & $\begin{array}{l}20 \\
0\end{array}$ & $\begin{array}{l}0.49 \\
0\end{array}$ & -0.009 & 0.010 & $\begin{array}{l}0.99 \\
3\end{array}$ & -0.007 & 0.010 & $\begin{array}{l}0.48 \\
9\end{array}$ & -0.031 & 0.008 & $\begin{array}{l}1.99 \\
0\end{array}$ & -0.012 & 0.014 \\
\hline & & & & $\begin{array}{l}40 \\
0 \\
\end{array}$ & $\begin{array}{l}0.49 \\
0\end{array}$ & -0.013 & 0.006 & $\begin{array}{l}0.99 \\
7 \\
\end{array}$ & -0.003 & 0.010 & $\begin{array}{l}0.49 \\
0\end{array}$ & -0.067 & 0.000 & $\begin{array}{l}1.99 \\
0\end{array}$ & -0.016 & 0.010 \\
\hline & & & & 25 & $\begin{array}{l}0.49 \\
0\end{array}$ & -0.010 & 0.010 & $\begin{array}{l}0.99 \\
6\end{array}$ & -0.004 & 0.010 & $\begin{array}{l}0.87 \\
8\end{array}$ & -0.031 & 0.009 & $\begin{array}{l}1.49 \\
0\end{array}$ & -0.013 & 0.014 \\
\hline & & & & 40 & $\begin{array}{l}0.48 \\
9\end{array}$ & -0.011 & 0.010 & $\begin{array}{l}0.99 \\
8\end{array}$ & -0.002 & 0.010 & $\begin{array}{l}0.90 \\
4\end{array}$ & -0.036 & 0.009 & $\begin{array}{l}1.48 \\
9\end{array}$ & -0.014 & 0.014 \\
\hline 0.5 & 1 & 1 & 1.5 & $\begin{array}{l}10 \\
0\end{array}$ & $\begin{array}{l}0.48 \\
8\end{array}$ & -0.012 & 0.009 & $\begin{array}{l}1.00 \\
6\end{array}$ & 0.006 & 0.010 & $\begin{array}{l}0.93 \\
8\end{array}$ & -0.062 & 0.006 & $\begin{array}{l}1.48 \\
8\end{array}$ & -0.015 & 0.013 \\
\hline & & & & $\begin{array}{l}20 \\
0\end{array}$ & $\begin{array}{l}0.48 \\
9\end{array}$ & -0.011 & 0.008 & $\begin{array}{l}1.01 \\
5\end{array}$ & 0.015 & 0.009 & $\begin{array}{l}0.96 \\
4\end{array}$ & -0.096 & 0.005 & $\begin{array}{l}1.48 \\
9\end{array}$ & -0.014 & 0.012 \\
\hline & & & & $\begin{array}{l}40 \\
0 \\
\end{array}$ & $\begin{array}{l}0.49 \\
3 \\
\end{array}$ & -0.007 & 0.004 & $\begin{array}{l}1.02 \\
2 \\
\end{array}$ & 0.022 & 0.008 & $\begin{array}{l}0.96 \\
9 \\
\end{array}$ & -0.122 & 0.010 & $\begin{array}{l}1.49 \\
3 \\
\end{array}$ & -0.010 & 0.008 \\
\hline & & & & 25 & $\begin{array}{l}0.49 \\
0\end{array}$ & -0.010 & 0.010 & $\begin{array}{l}2.99 \\
0\end{array}$ & -0.010 & 0.010 & $\begin{array}{l}0.92 \\
5\end{array}$ & -0.011 & 0.010 & $\begin{array}{l}1.49 \\
0\end{array}$ & -0.013 & 0.014 \\
\hline & & & & 40 & $\begin{array}{l}0.49 \\
0\end{array}$ & -0.010 & 0.010 & $\begin{array}{l}2.99 \\
0\end{array}$ & -0.010 & 0.010 & $\begin{array}{l}0.96 \\
2\end{array}$ & -0.012 & 0.010 & $\begin{array}{l}1.49 \\
0\end{array}$ & -0.013 & 0.014 \\
\hline 0.5 & 3 & 1 & 1.5 & $\begin{array}{l}10 \\
0\end{array}$ & $\begin{array}{l}0.48 \\
9\end{array}$ & -0.011 & 0.010 & $\begin{array}{l}2.99 \\
1\end{array}$ & -0.009 & 0.010 & $\begin{array}{l}0.97 \\
6\end{array}$ & -0.024 & 0.009 & $\begin{array}{l}1.48 \\
9\end{array}$ & -0.014 & 0.014 \\
\hline & & & & $\begin{array}{l}20 \\
0\end{array}$ & $\begin{array}{l}0.49 \\
2\end{array}$ & -0.008 & 0.009 & $\begin{array}{l}2.99 \\
2\end{array}$ & -0.008 & 0.010 & $\begin{array}{l}0.98 \\
8\end{array}$ & -0.038 & 0.008 & $\begin{array}{l}1.49 \\
2\end{array}$ & -0.011 & 0.013 \\
\hline & & & & $\begin{array}{l}40 \\
0 \\
\end{array}$ & $\begin{array}{l}0.49 \\
2 \\
\end{array}$ & -0.008 & 0.004 & $\begin{array}{l}2.99 \\
4 \\
\end{array}$ & -0.006 & 0.010 & $\begin{array}{l}0.98 \\
9 \\
\end{array}$ & -0.075 & 0.001 & $\begin{array}{l}1.49 \\
2 \\
\end{array}$ & -0.011 & 0.008 \\
\hline & & & & 25 & $\begin{array}{l}0.48 \\
9\end{array}$ & -0.011 & 0.010 & $\begin{array}{l}2.99 \\
3\end{array}$ & -0.007 & 0.010 & $\begin{array}{l}2.90 \\
1\end{array}$ & -0.025 & 0.010 & $\begin{array}{l}1.98 \\
9\end{array}$ & -0.014 & 0.014 \\
\hline & & & & 40 & $\begin{array}{l}0.49 \\
0\end{array}$ & -0.009 & 0.010 & $\begin{array}{l}2.99 \\
4\end{array}$ & -0.006 & 0.010 & $\begin{array}{l}2.92 \\
7\end{array}$ & -0.029 & 0.010 & $\begin{array}{l}1.99 \\
0\end{array}$ & -0.013 & 0.014 \\
\hline 0.5 & 3 & 3 & 2 & $\begin{array}{l}10 \\
0\end{array}$ & $\begin{array}{l}0.48 \\
9\end{array}$ & -0.011 & 0.009 & $\begin{array}{l}2.99 \\
7\end{array}$ & -0.003 & 0.010 & $\begin{array}{l}2.96 \\
2\end{array}$ & -0.038 & 0.009 & $\begin{array}{l}1.98 \\
9\end{array}$ & -0.014 & 0.013 \\
\hline & & & & $\begin{array}{l}20 \\
0\end{array}$ & $\begin{array}{l}0.49 \\
5\end{array}$ & -0.005 & 0.008 & $\begin{array}{l}3.00 \\
6\end{array}$ & 0.006 & 0.009 & $\begin{array}{l}2.97 \\
1\end{array}$ & -0.073 & 0.001 & $\begin{array}{l}1.99 \\
5\end{array}$ & -0.009 & 0.012 \\
\hline & & & & $\begin{array}{l}40 \\
0\end{array}$ & $\begin{array}{l}0.49 \\
6\end{array}$ & -0.004 & 0.003 & $\begin{array}{l}3.00 \\
5\end{array}$ & 0.005 & 0.010 & $\begin{array}{l}2.97 \\
5\end{array}$ & -0.099 & 0.029 & $\begin{array}{l}1.99 \\
6\end{array}$ & -0.007 & 0.007 \\
\hline
\end{tabular}

Also, a real data set is used to discuss that the MOGIW can be a good ness of fit model to data comparing with several known distributions. These distributions are Marshall-Olkin extended inverse Weibull (MOEIW), inverse Weibull (IW), Fréchet (F), inverse Rayleigh (IR) and inverse exponential (IE). Using the MATHCAD software, the parameters of the MOGIW distribution are estimated via MLE method as follows: First, the MLEs and the corresponding standard errors of the parameters are reported. Second, the formal goodness-of-fit tests including Akaike information criterion $(A I C=-2 \ln L+2 p)$, consistent Akaike information criterion 
$\left(C A I C=-2 \ln L+\frac{2 p n}{n-p-1}\right)$, Bayesian information criterion $(B I C=-2 \ln L+p \ln n)$ and Hannan-Quinn

information criterion $(H Q I C=-2 \ln L+p \ln (\ln n))$ are applied to verify which distribution fits better the real data set. where $\mathrm{p}$ is the number of the model parameters and $\mathrm{n}$ is the sample size. In general, the smaller values of these statistics is the better fit to data. The real data set was originally reported by (Bjerkedal 1960), which represents two treatments of guinea pigs. Each treatment divided into 6 groups of 72 guinea pigs of animals which observed for two years. Table (2) gives a descriptive summary for these data.

Table 2.72 observations for guinea pigs of animals.

\begin{tabular}{llllllllllllllllll}
\hline 12 & 15 & 22 & 24 & 24 & 32 & 32 & 33 & 34 & 38 & 38 & 43 & 44 & 48 & 52 & 53 & 54 & 54 \\
55 & 56 & 57 & 58 & 58 & 59 & 60 & 60 & 60 & 60 & 61 & 62 & 63 & 65 & 65 & 67 & 68 & 70 \\
70 & 72 & 73 & 75 & 76 & 76 & 81 & 83 & 84 & 85 & 87 & 91 & 95 & 96 & 98 & 99 & 109 & 110 \\
121 & 127 & 129 & 131 & 143 & 146 & 146 & 175 & 175 & 211 & 233 & 258 & 258 & 263 & 297 & 341 & 341 & 376 \\
\hline
\end{tabular}

Table (3) shows that the MLEs estimates for the parameters for the MOGIW distribution and their sub-models by using equations, (17) - (20). We notice that, the proposed model presents the best model among all others to fit this data because the MOGIW presents the smallest values of the statistics AIC, BIC, CAIC and HQIC. Also, some statistics such Kolmogrov-Smirnov (K-S), Anderson-Darling (A) and Cramér-von Mises (W) are used. The values of these tests lead to that the proposed model presents the best model among all others because the proposed model is the smallest values of all statistics. While, the p-value of the MOGIW distribution is the greater value than others.

Table 3. (K-S), (A), (W), AIC, BIC, CAIC and HQIC statistics for data.

\begin{tabular}{ccccccccc}
\hline Distribution & K-S & p-value & A & W & AIC & BIC & CAIC & HQIC \\
\hline MOGIW & 0.07112 & 0.8575 & 0.6081 & 0.10376 & 111.714 & 110.71 & 112.766 & 113.952 \\
MOEIW & 0.07627 & 0.8445 & 0.6341 & 0.10522 & 153.598 & 152.595 & 154.651 & 154.471 \\
IW & 0.1318 & 0.1381 & 1.5177 & 0.2538 & 289.691 & 288.687 & 290.743 & 291.375 \\
F & 0.6398 & $8.95^{*} 10^{\wedge}-27$ & 44.042 & 9.6086 & 121.867 & 132.583 & 122.920 & 123.892 \\
IR & 0.2396 & $4.88^{*} 10^{\wedge}-4$ & 6.5639 & 1.2661 & 120.594 & 129.166 & 121.283 & 121.885 \\
IE & 0.1864 & 0.0128 & 4.6425 & 0.8379 & 118.700 & 125.130 & 119.107 & 120.225 \\
\hline
\end{tabular}

\section{Conclusion}

The well-known inverse Weibull distribution, is extended by introducing one extra shape parameters, thus defining the Marshal Olikin Generalized Inverse Weibull (MOGIW) distribution having a broader class of hazard rate and density functions. This is achieved by taking the Inverse Weibull cdf as the baseline cumulative distribution of the Marshal Olikin family. A detailed study on the statistical properties of the new model is presented. The MOGIW distribution includes as special sub-models as Marshal Olikin Inverse Weibull (MOIW), exponentiated inverse Weibull (EIW), inverse Weibull (IW), Fréchet (F), inverse Rayleigh (IR) and inverse exponential (IE) distributions. Estimation of MOGIW distribution parameters is obtained by maximum likelihood and least square methods as well as the observed information matrix and asymptotic confidence intervals are discussed. An application to a real data set indicates that the new model fits data than the principal sub-models.

\section{References}

Alice, T., \& Jose, K. (2005). Marshall-Olkin logistic processes. STARS Int. J., 6, 1-11.

Bennette, S. (1983). Log-Logistic Regression Models for Survival Data. Journal of the Royal Statistical Society Applied Statistics, 32, 165-171. Retrieved from https://www.jstor.org/stable/2347295

Bjerkedal, T. (1960). Acquisition of resistance in guinea pigs infected with different doses of virulent tubercle bacilli. Am. J. Hyg., 72, 130-148. https://doi.org/10.1093/oxfordjournals.aje.a120129

Cordeiro G., \& Lemonte A. (2013). On the Marshall-Olkin extended weibull distribution. Stat. Papers, 54, 333- 
353. https://doi.org/10.1007/s00362-012-0431-8.

Drapella, A. (1993). Complementary Weibull Distribution: Unknown or Just Forgotten. Quality and Reliability Engineering International, 9, 383-385. https://doi.org/10.1002/qre.4680090426

Felipe, R., Edwin, M., \& Gauss, M. (2011). The generalized inverse Weibull distribution. Statistical Papers, 52, 591- 619. https://doi.org/10.1007/s00362-009-0271-3

Flaih, A., Elsalloukh, H, Mendi, E., \& Milanova, M. (2012). The exponentiated inverse Weibull distribution. Appl Math Inf. Sci., 6, 167-171.

Ghitany, M. (2005). Marshall-Olkin extended pareto and its application. Int. J. Appl. Math., 18, 17-32.

Ghitany, M. Al-Awadhi, F., \& L. Alkhalfan, L (2007). Marshall-Olkin extended lomax distribution and its application to censored data. Commun. Stat. Theory Methods, 36, 1855-1866. https://doi.org/10.1080/03610920601126571

Ghitany, M., Al-Hussaini, E., \& Al-Jarallah, R. (2005). Marshall-Olkin extended Weibull distribution and its application to censored data. J. Appl. Stat., 32, 1025-1034. https://doi.org/10.1080/02664760500165008

Gui, W. (2013). Marshall-Olkin power log-normal distribution and its applications to survival data. Int. J. Stat. Probab., 2, 63-72. http://dx.doi.org/10.5539/ijsp.v2n1p63

Jose, K., \& Krishna, E. (2011). Marshall-Olkin extended uniform distribution. Probab. Stat. Optim., 4, 78-88.

Kundu, D., \& Howlader, H. (2010). Bayesian Inference and Prediction of the Inverse Weibull Distribution for Type-II Censored Data. Computational Statistics and Data Analysis, 54, 1547-1558. https://doi.org/10.1016/j.csda.2010.01.003

Langlands, A., Pocock, S., Kerr, G., \& Gore, M. (1979). Long Term Survival of Patients with Breast Cancer: A Study of Curability of the Disease. British Medical Journal, 1247-1251. https://www.ncbi.nlm.nih.gov/pmc/articles/PMC1596940/

Marshall, A., \& Olkin I. (1997). A new method for adding a parameter to a family of distributions with applications to the exponential and Weibull families. Biometrika,84, 641-652.https://doi.org/10.1093/biomet/84.3.641

Mudholkar, G., \& Kollia, G. (1994). Generalized Weibull Family: A Structural Analysis. Communications in Statistics - Theory and Methods, 23, 1149-1171. https://doi.org/10.1080/03610929408831309

Okasha, H., \& Kayid, M. (2016). A new family of Marshall-Olkin extended generalized linear exponential distribution. J. Comput. Appl. Math., 296, 576-592. https://doi.org/10.1016/j.cam.2015.10.017

Okasha, H., El-Baz, A., Tarabia, A., \& Basheer, A. (2017). Extended inverse Weibull distribution with reliability application. Journal of the Egyptian Mathematical Society, 25, 343-349. https://doi.org/10.1016/j.joems.2017.02.006

Pararai, M., Warahena, G., \& Oluyede, B. (2014). A new class of generalized inverse Weibull distribution with applications. J. Appl. Math., 4, 17-35.

Swain, J., Venkatraman, S., \& Wilson, J. (1988). Least squares estimation of distribution function in Johnson's translation system. Journal of Statistical Computation and Simulation,29,271-297. https://doi.org/10.1080/00949658808811068

\section{Copyrights}

Copyright for this article is retained by the author(s), with first publication rights granted to the journal.

This is an open-access article distributed under the terms and conditions of the Creative Commons Attribution license (http://creativecommons.org/licenses/by/4.0/). 